\title{
Calibrating the Dark Energy Survey: The Role of DA White Dwarfs
}

J.A. Smith, D.J. Gulledge (APSU); J.M. Robertson (COMPASS Science Communication); M.B. Fix (STScl); S. Charbonnier (Ecole Polytechnique); D.L. Tucker, W. Wester, S.S. Allam (Fermilab); P-E. Tremblay (U. Warwick); G. Narayan (STScl); J. Marriner, B. Yanny, K. Herner (Fermilab); J. Lasker (U. Chicago)

$21^{\text {st }}$ European White Dwarf Workshop 2018

Austin, Texas 23 July 2018

This document was prepared by [DES Collaboration] using the resources of the Fermi National Accelerator Laboratory (Fermilab), a U.S. Department of Energy, Office of Science, HEP User Facility. Fermilab is managed by Fermi Research Alliance, LLC (FRA), acting under Contract No. DE-AC02-07CH11359. 


\section{Blanco + DECam}

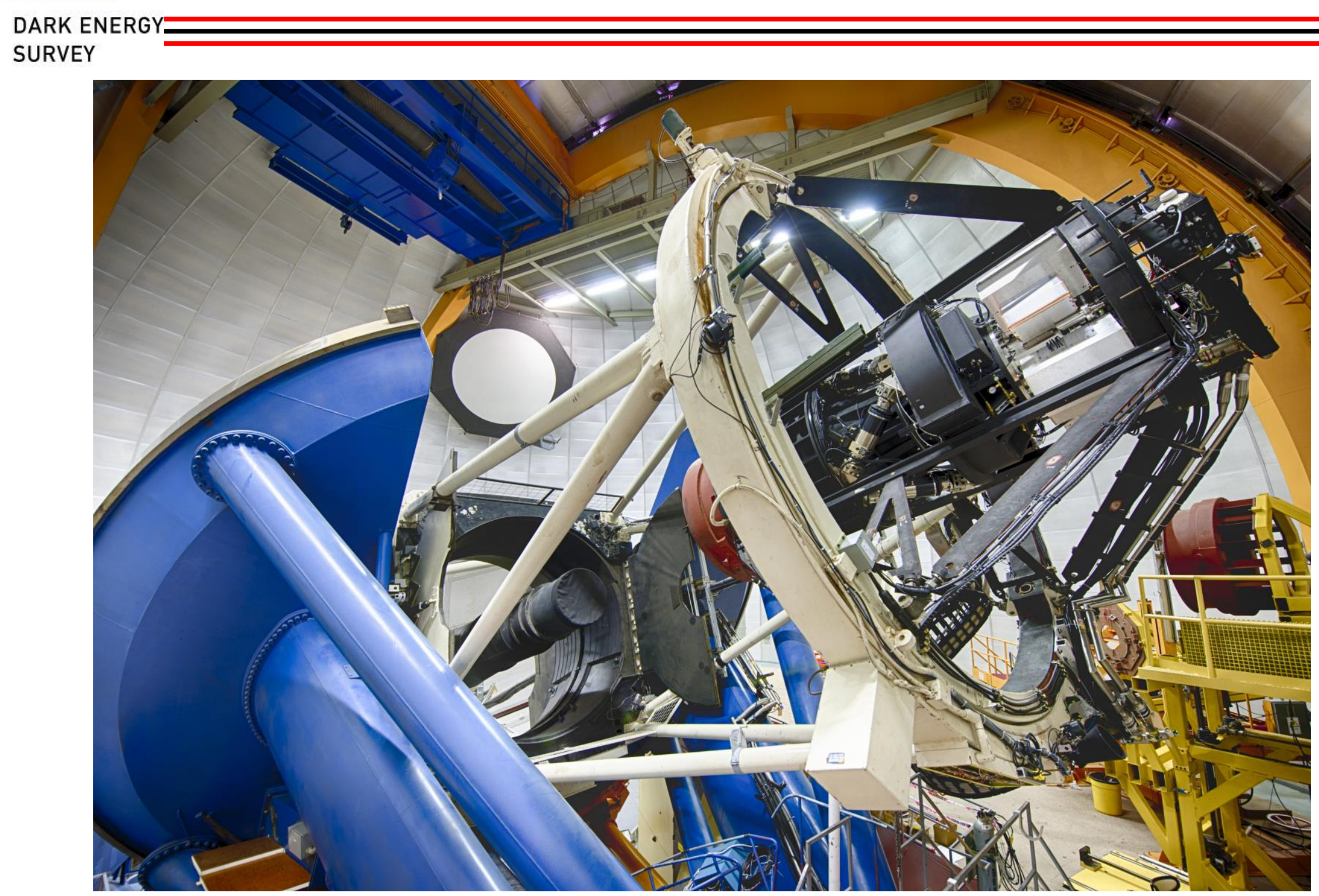




\section{Ancillary Hardware}

DARK ENERGY SURVEY

\section{SLAC}

$10 \mu \mathrm{m}$ all-sky

camera
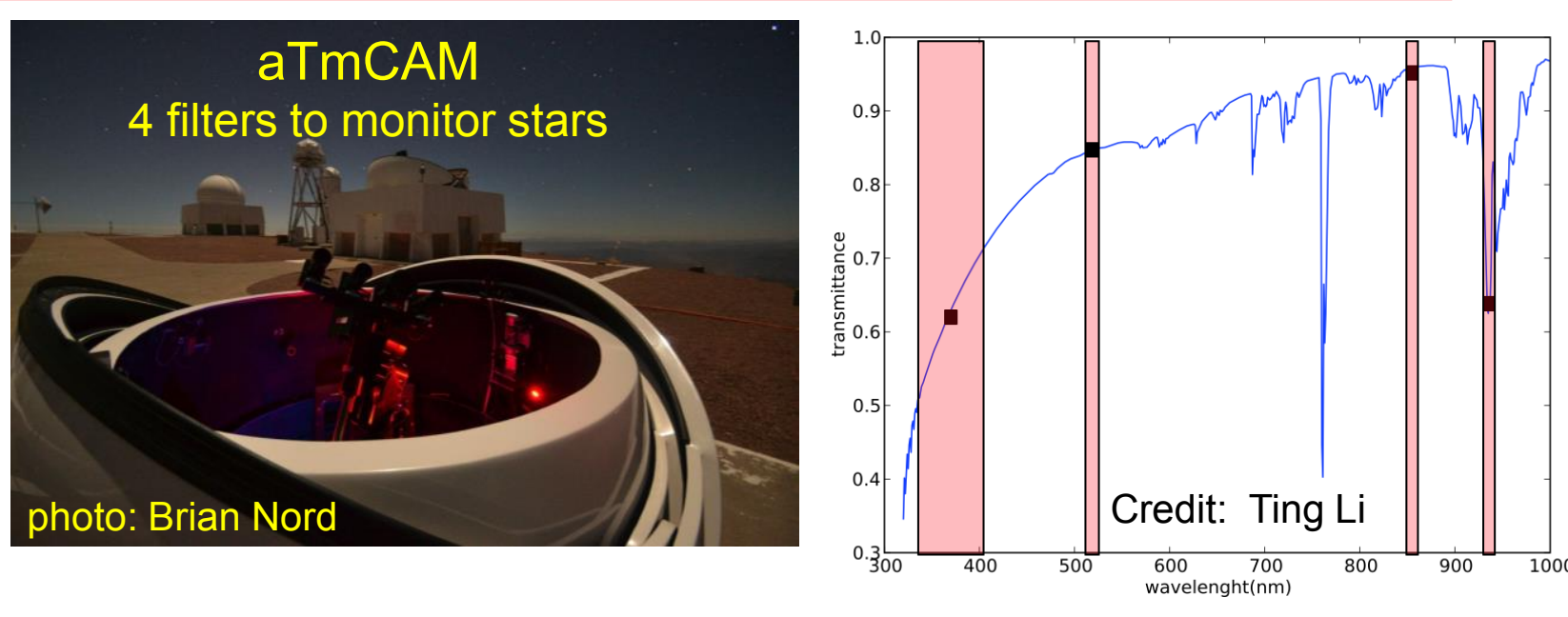

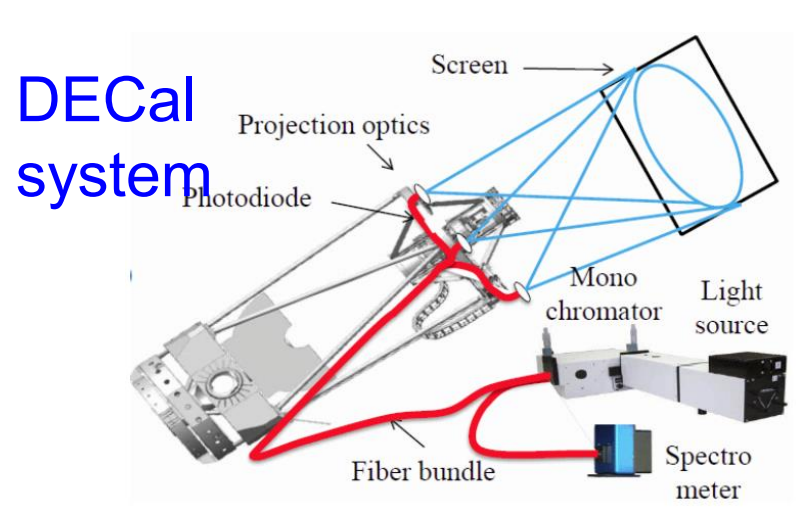

Marshall et al. 2013

Slide Credit: William Wester

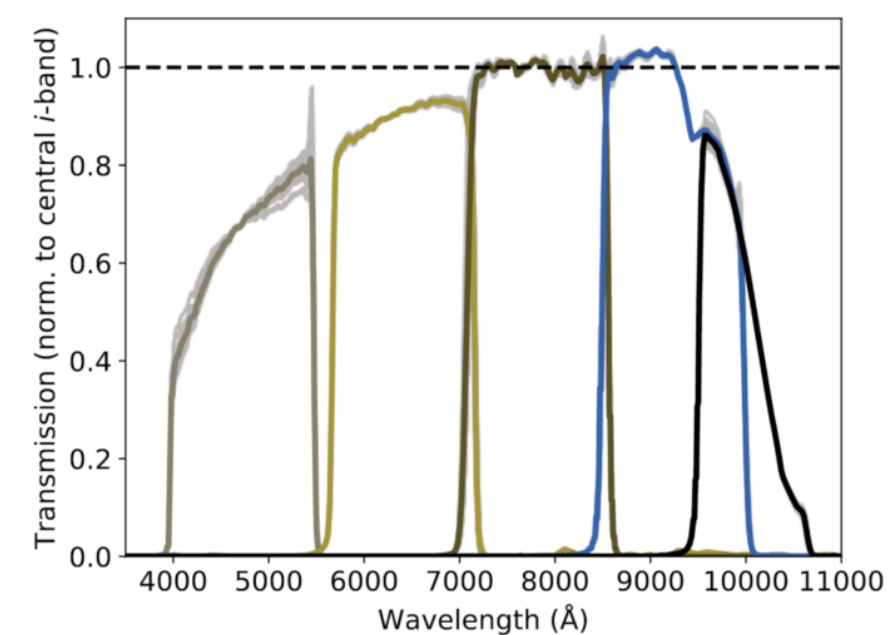

GPS monitor

(to measure PWV)

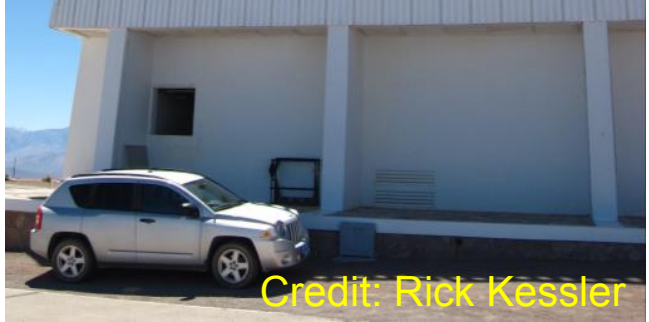




\section{The Dark Energy Survey (DES)}

\section{DARK ENERGY}

SURVEY

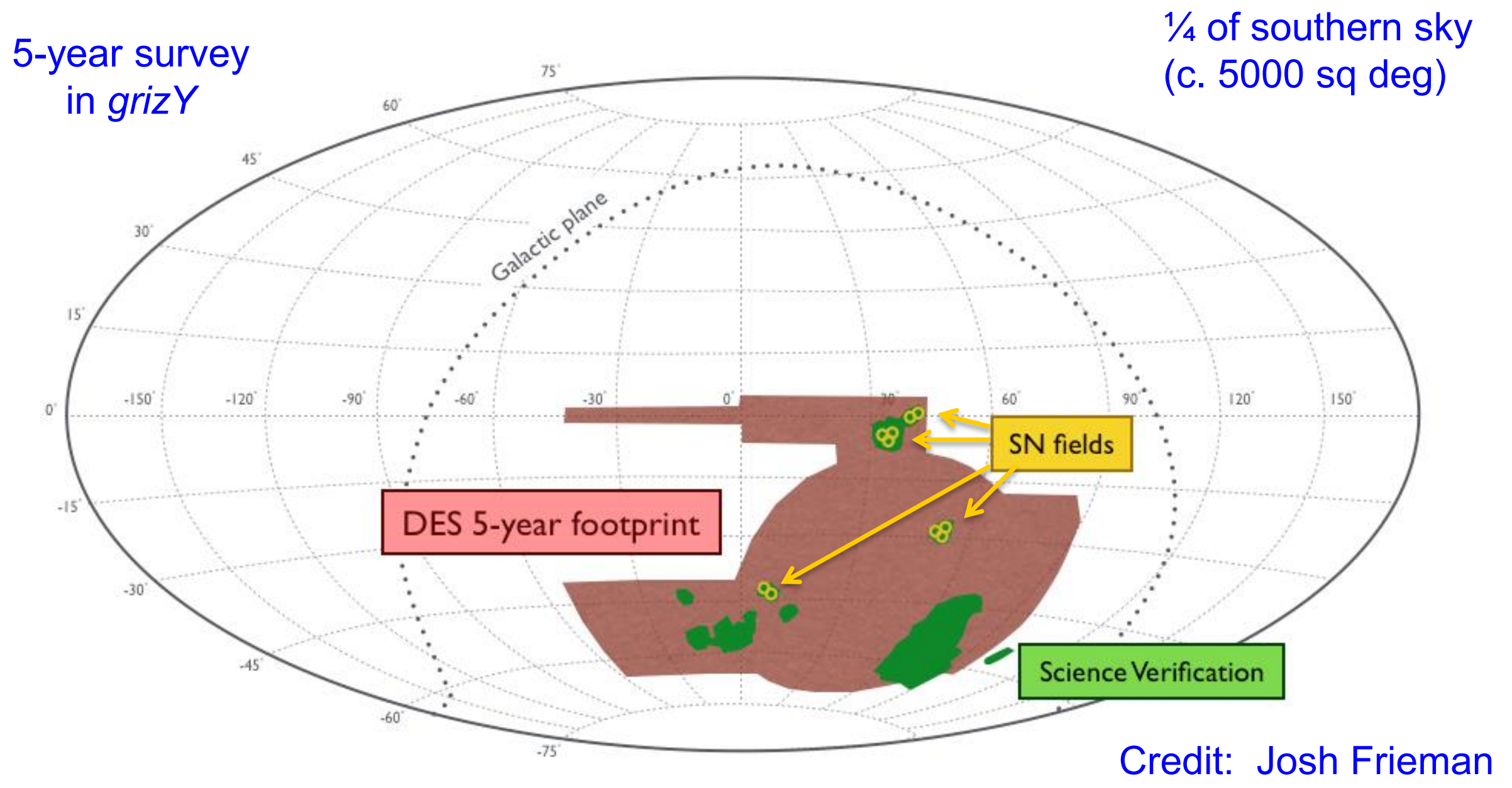




\section{DARK ENERGY}

SURVEY

Photometry 



\section{Many parts to calibration}

DARK ENERGY SURVEY

DES Photometric Calibrations Flow Diagram (v4.1)

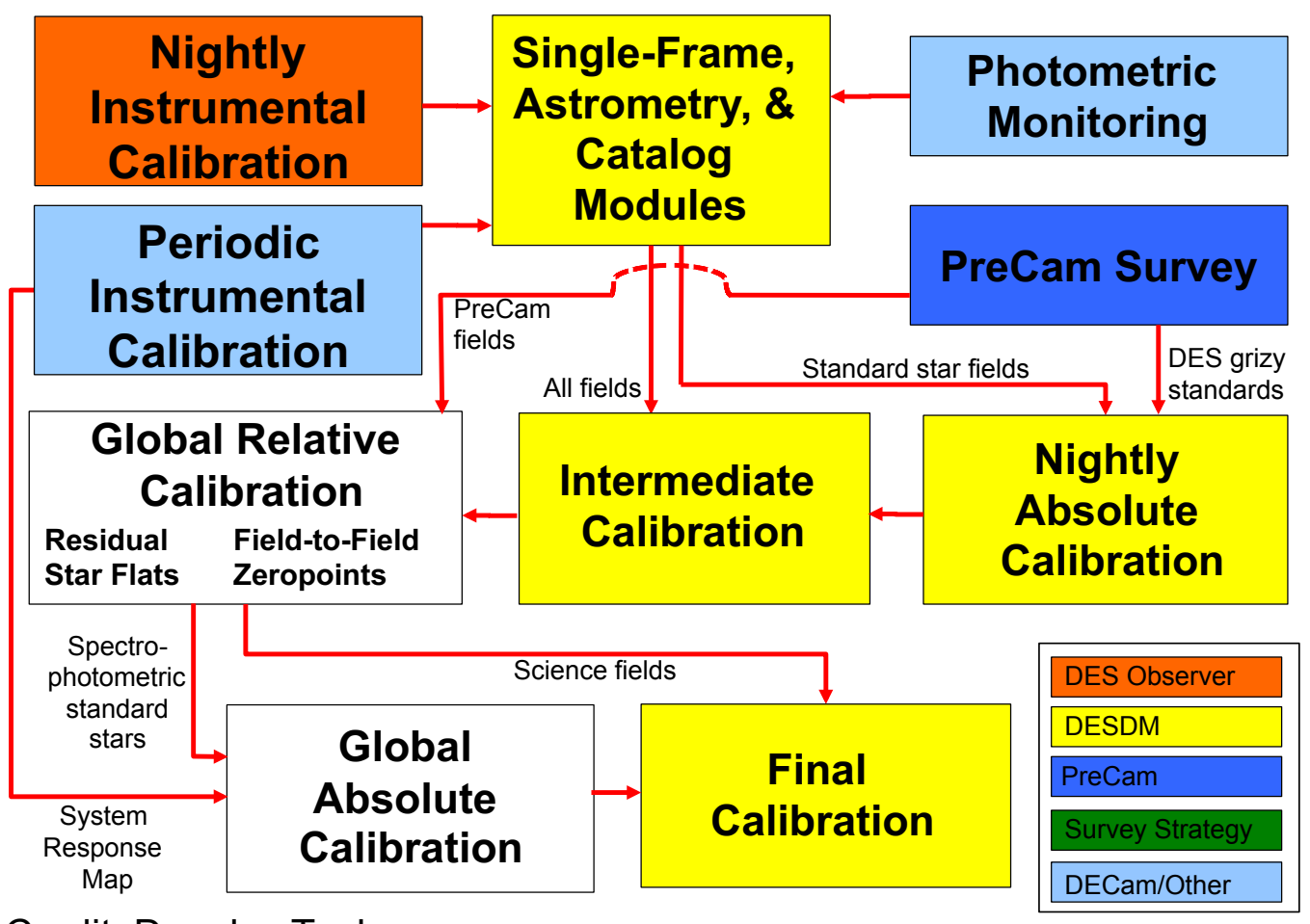

- Nightly

- Bias, darks, flat fields

- Periodic

- Spectral scanning

- Transfer function

- Star flats

- Monitoring

- Atmosphere aTmCAM / GPS

- Cloud cover

- Absolute scale

- White Dwarf

- CALSPEC standards 


\section{DES Photometry: SV, Y1, (Y3) PGCM}

DARK ENERGY SURVEY

- Photometric Global Calibration Module (PGCM)

- Observe nightly standards to create a sparse gridwork of tertiary standards.

- Use overlapping exposures to tie DES photometry to tertiary network.

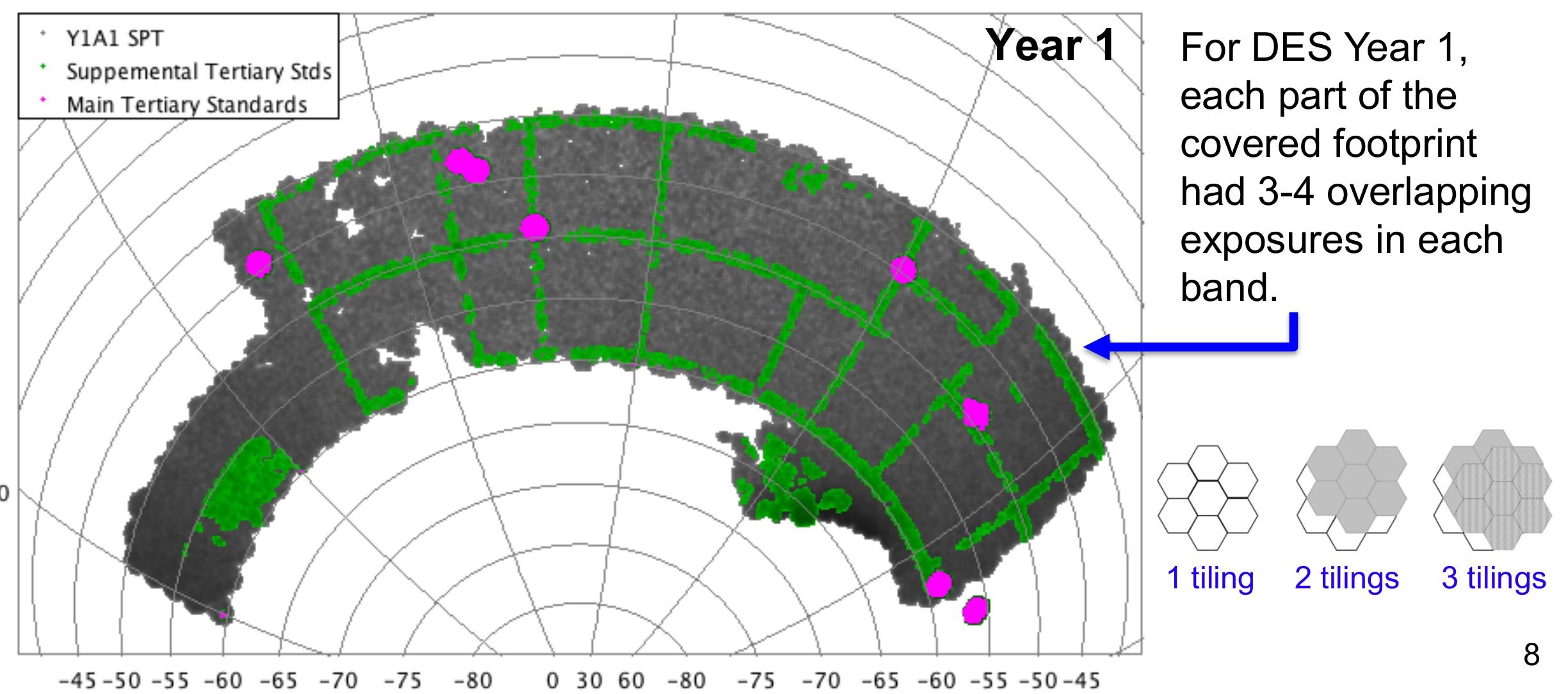




\section{Photometric Equation}

DARK ENERGY

SURVEY

$m_{\text {inst }}-m_{\text {std }}=a_{n}+b_{n} x\left(\right.$ stdColor - stdColor $\left.{ }_{0}\right)+k X \quad$ Credit: Douglas Tucker

- $m_{\text {inst }}$ is the instrumental magnitude, $m_{\text {inst }}=-2.5 \log$ (counts/sec) (input)

- $m_{\text {std }}$ is the standard ("true") magnitude of the standard star (input)

- $a_{n}$ is the photometric zeropoint for CCD $n(n=1-62)$ (output)

- $k$ is the first-order extinction (input/output)

- $X$ is the airmass (input)

- $b_{n}$ is the instrumental color term coefficient for CCD $n(n=1-62)$ (input/output)

- stdColor is a color index, e.g., (g-r) (input)

- $s t d C o l o r_{0}$ is a constant (a fixed reference value for that passband) (input)

- DES calibrations will be in the DECam natural system, but there may be variations from $C C D$ to $C C D$ within the DECam focal plane or over time.

$\mathbf{a}_{\mathbf{n}}$ : Zeropoints and uncertainty as a function of exposure and CCD number 


\section{General Idea for Absolute Calibration with DA WDs}

\section{DARK ENERGY}

\section{SURVEY}

- Compare the synthetic magnitudes to the measured magnitudes of one or more DA WDs observed by the DECam.

- The differences are the zeropoint offsets needed to tie the DES mags to an absolute flux in physical units (e.g., ergs $\left.\mathrm{s}^{-1} \mathrm{~cm}^{-2} \AA^{-1}\right)$.

- For the synthetic photometry, the fit model spectra of the white dwarfs are generally

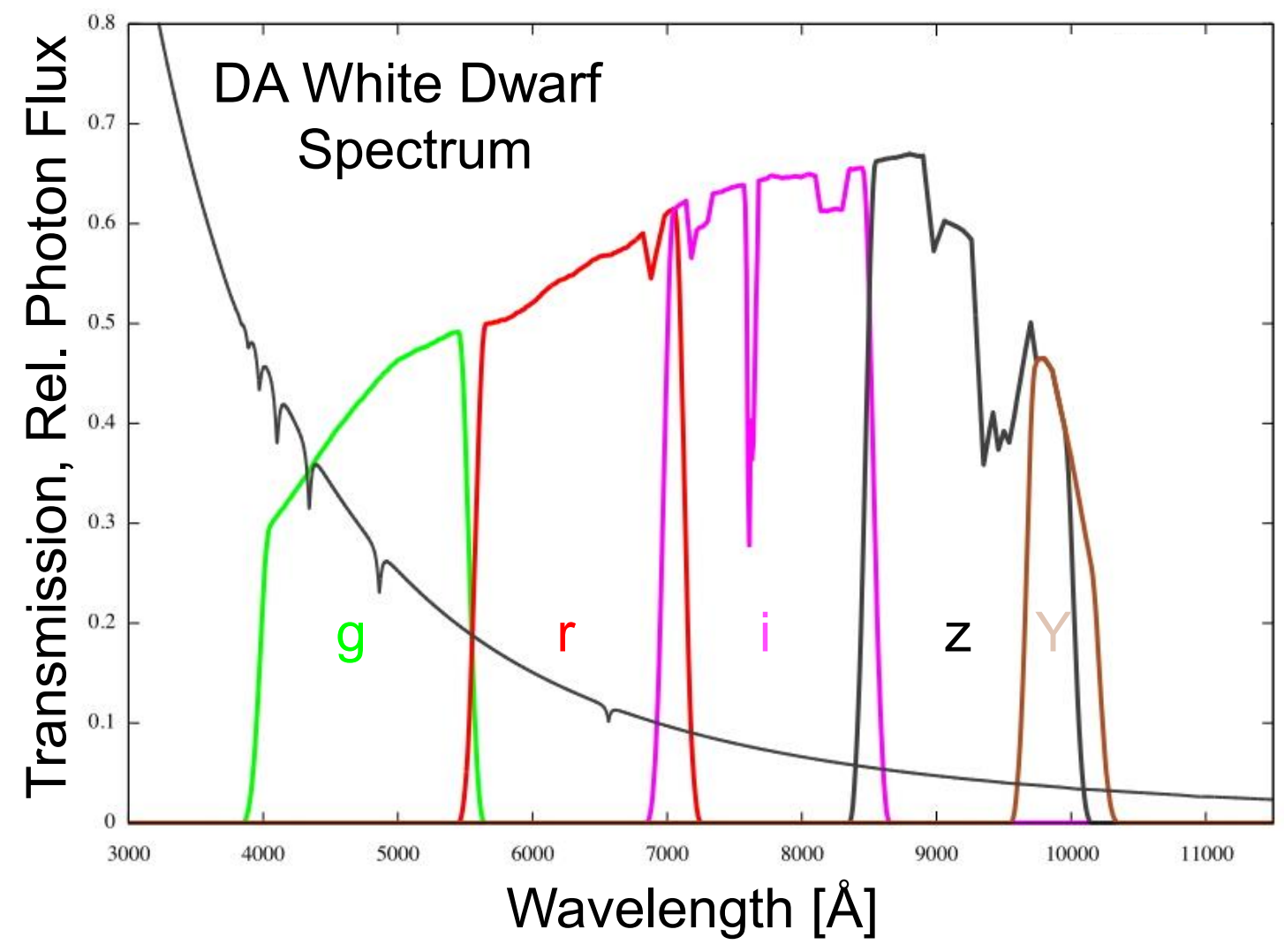
used.

Plan: establish a "Golden Sample" of 30-100 wellcalibrated DA white dwarfs across the DES footprint. 


\section{FGCM Absolute Calibration}

(also relevant to other calibration methods)

\section{DARK ENERGY}

SURVEY

- Three CALSPEC standards in DES footprint. Only one is a faint standard. FGCM has absolute scale set to C26202.

- DES DR1: 3-5 mmag uncertainty, relative to $\mathrm{C} 26202$.

- Multi-year program of identifying white dwarf candidates $(\sim 100)$, obtaining spectra, and performing model fits giving synthetic spectra.

SOAR-4m Spectra of Candidate DA WDs

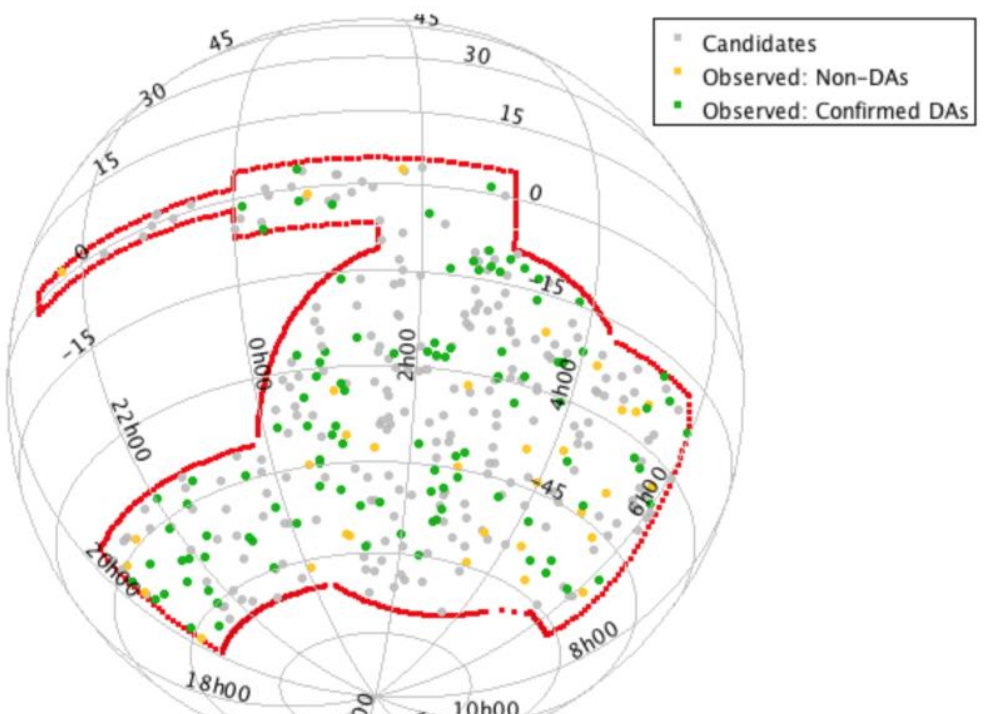

Synthetic photometry can be compared with observed mags.

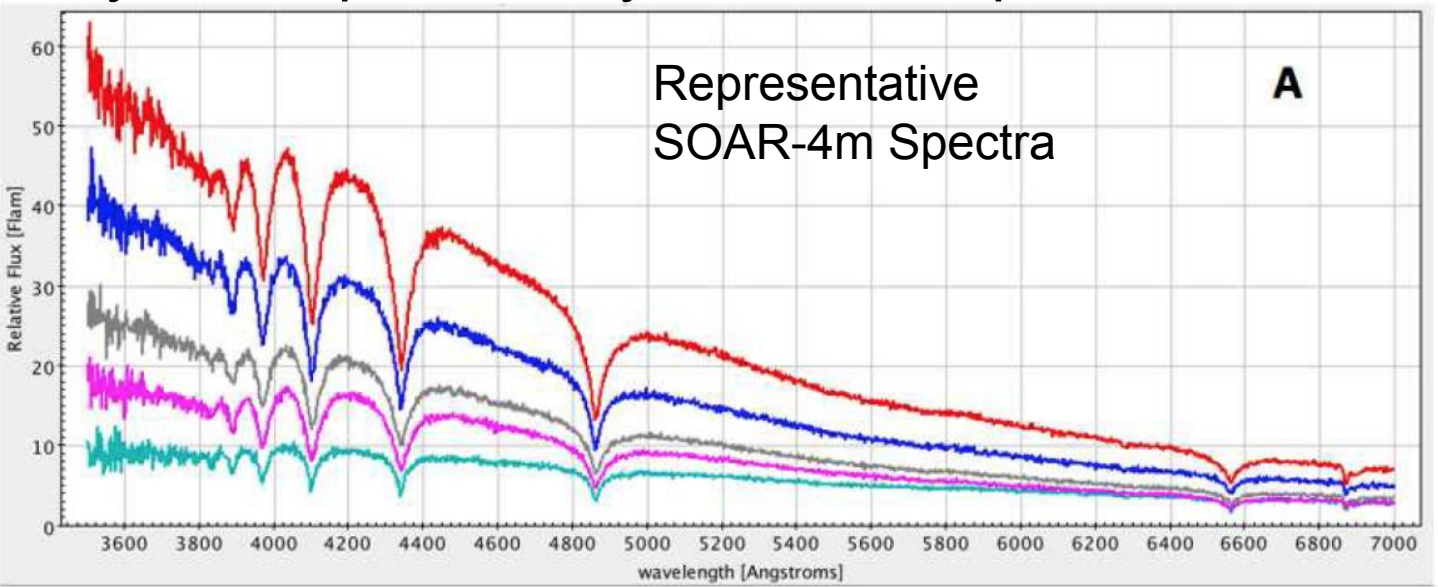

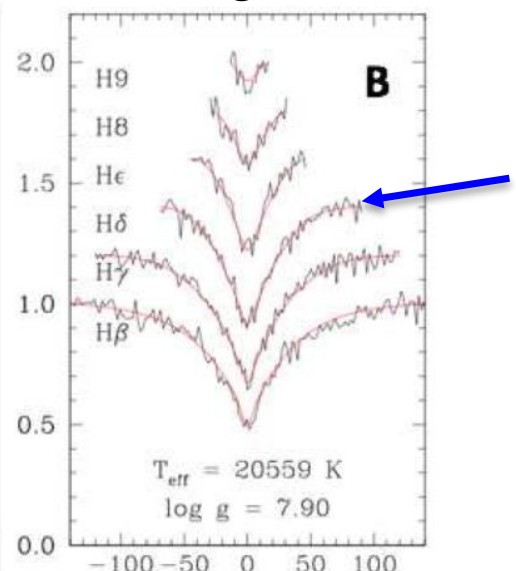

$\Delta \lambda(\AA)$
DA WD atm. model fits

(P.-E. Tremblay)

(Also using G. Narayan's WDmodel)

Slide credit: William Wester 


\section{A Representative Model Fit (SOAR-4m spectrum)}

DARK ENERGY SURVEY

Credit: Pier-Emmanuel Tremblay
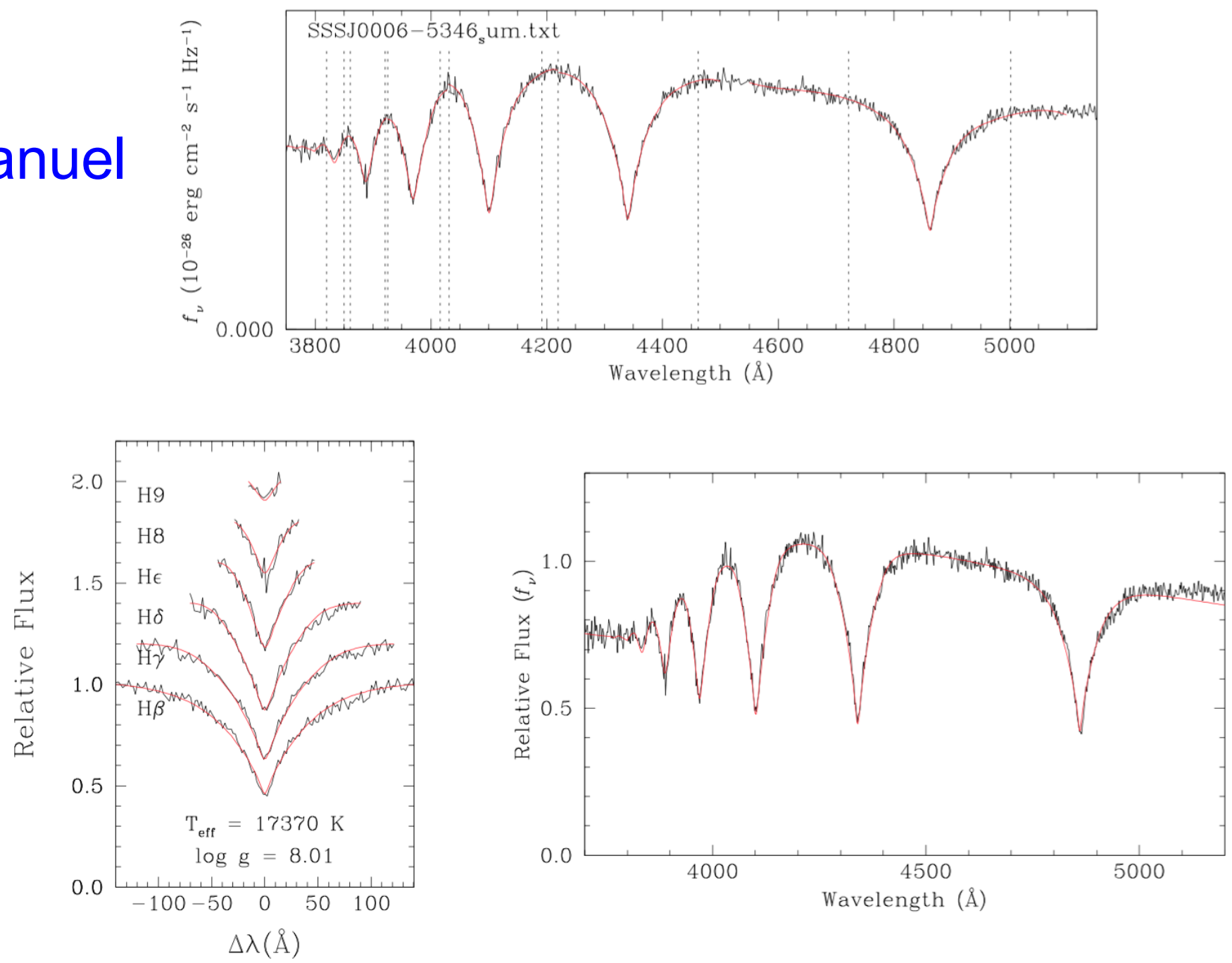


\section{A Representative Model Fit: Tremblay vs. Narayan codes}

DARK ENERGY SURVEY

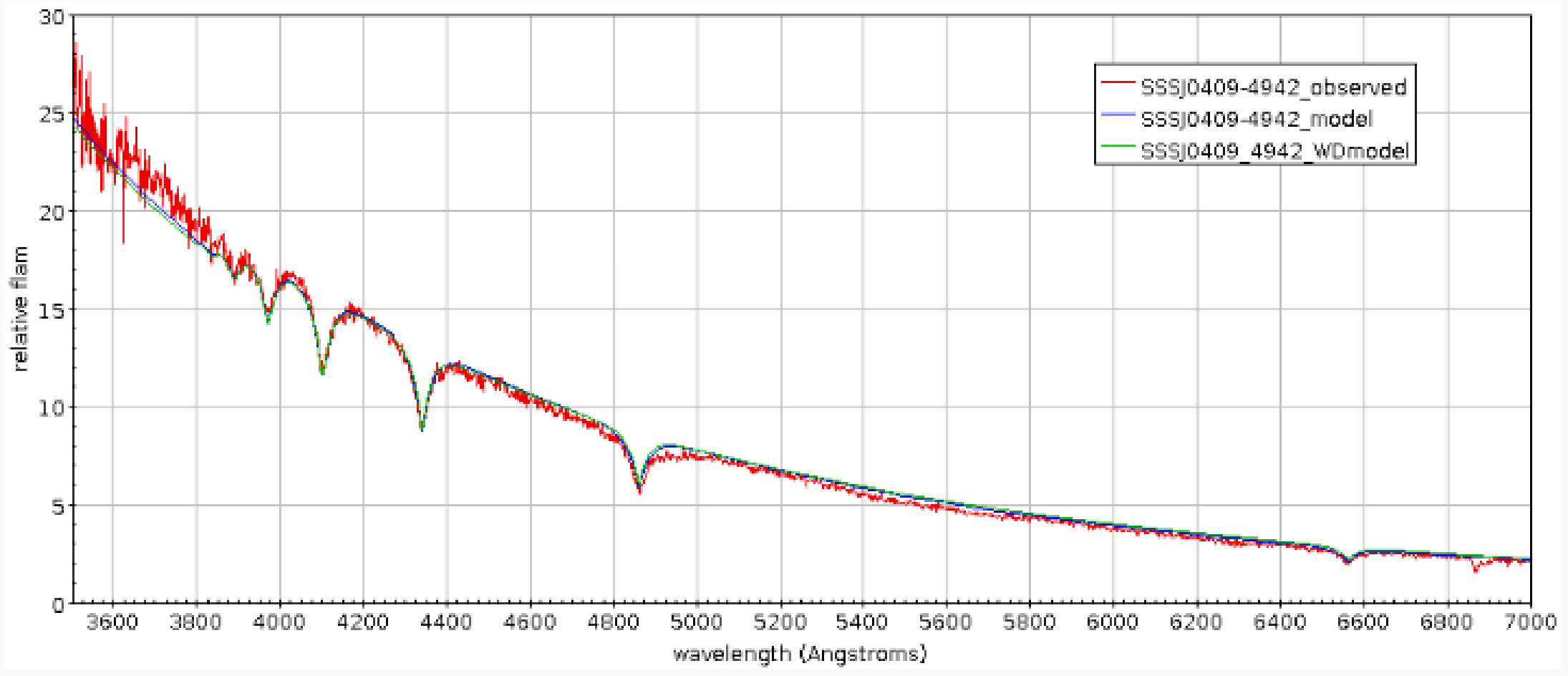




\section{What about Interstellar Reddening?}

DARK ENERGY SURVEY

One of the highest amounts of line-of-sight $E(B-V)$ 's for our current sample.

But is this WD within the Local Bubble? Behind the screen of MW dust? Or embedded within it?

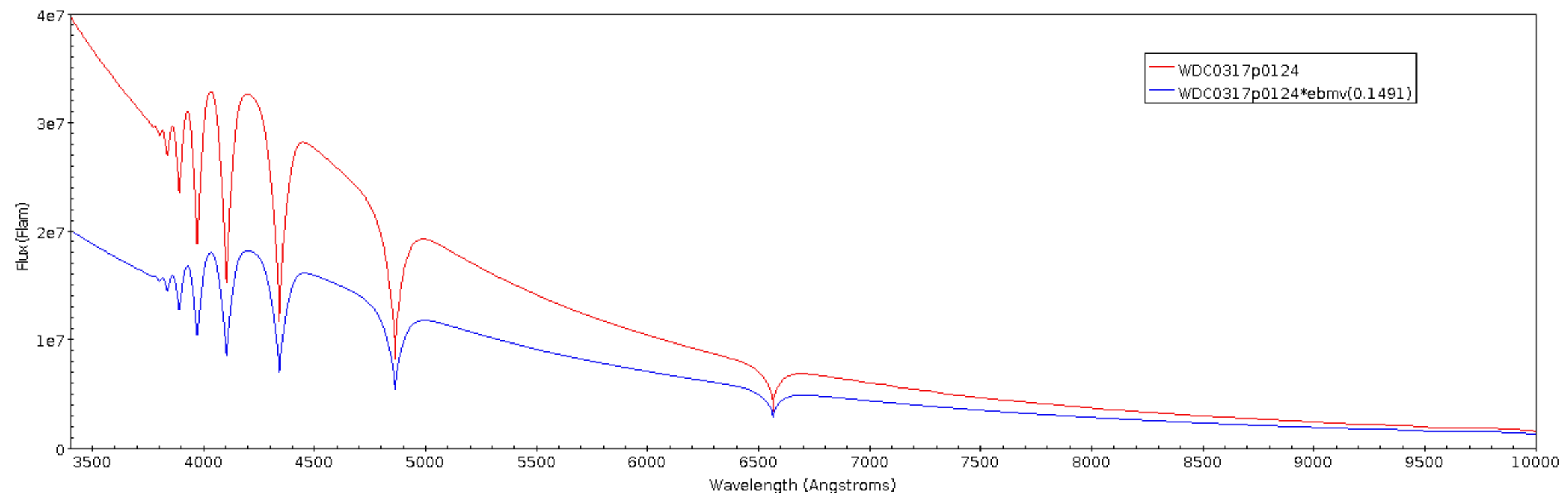

Credit: Deborah Gulledge 


\section{Test Run by FNAL Group}

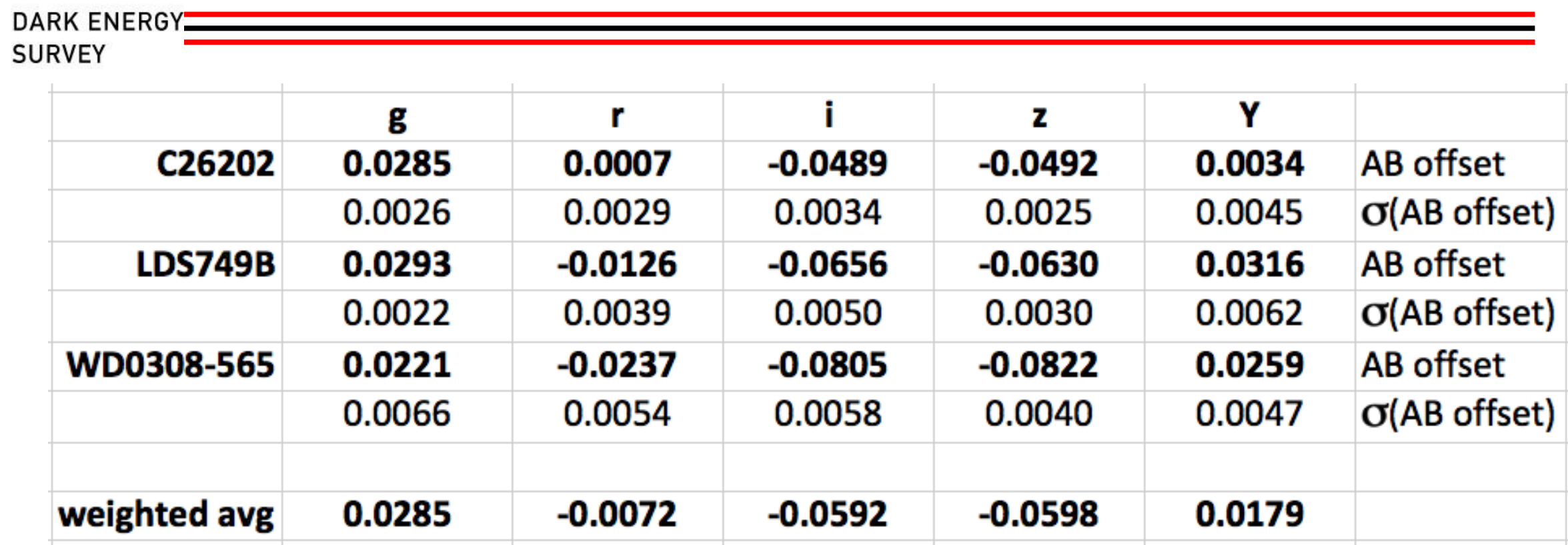

We had to be careful as the synthetic magnitude can be mis-calculated if the HST Spectra doesn't cover the full DES wavelength range.

Future study will be to look at chromatic effects (LDS749B and WD0308-565 are White Dwarfs, C26202 is a solar analog) and other ways to reduce the $\sigma$ (AB offset) or improve precision (check airmass of observation, for instance).

Bottom line: is that there are initial offsets that can be used to put the FGCM onto the $A B$ scale. Future work will improve the precision. 


\section{Current Spectroscopic Results $-1.5 \mathrm{~m}$}

DARK ENERGY:

SURVEY

- CTIO 1.5m:

- 154 total objects reduced:

- 25 were targeted for the WD program: 8 identified WDs

- Generally, they are too bright to use for DES calibration, but worked well as a training set.

- Paper in preparation: Gulledge et al. (2019?)

- See Smith Poster for some of these. 


\section{Current Spectroscopic Results}

DARK ENERGY

SURVEY

- SOAR: (SuperCosmos + ATLAS selections):

- 145 targets (so far): 11 DAs/ 12 DBs/ 2 magnetics/ 20 "other"

- APO - 3.5m: Mostly SDSS color selected

- 83 targets: $~ 75 \%$ DA/ 2-QSOs/ 1-CV/ Handful of DB/Other

- AAT - 4m: 32 spectra obtained. Still in reduction

- Magellan: 12 spectra (2016): 11 DAs

- Still working on 2017 data and waiting for 2018 night 


\section{Imaging Follow-up}

DARK ENERGY SURVEY

\section{CTIO-0.9m, WIYN-0.9m}

1. To obtain SDSS u-band photometry of Rowell \& Hambly sample (to use color selection to improve success rate)

2. To monitoring candidates for signs of variability.

Currently, 400 candidate white dwarfs have been imaged as part of the imaging follow-up program. 


\section{Conclusions}

DARK ENERGY SURVEY

- DES continues to evolve in its calibrations

- Advanced knowledge of the devices and readout

- External inputs for clouds and the atmosphere

- FGCM reaches $2 \%$ requirements in magnitudes

- With knowledge of SEDs, $0.5 \%$ color uncertainties and sub-1\% photometry has been achieved

- Work on absolute calibration continues and the white dwarf sample will have legacy with LSST etc. 